\title{
Seroprevalence and risk factors for Rickettsia felis exposure in dogs from Southeast Queensland and the Northern Territory, Australia
}

\author{
Sze-Fui Hii ${ }^{1{ }^{*}}$, Mohammad Y Abdad ${ }^{2 \dagger}$, Steven R Kopp ${ }^{1}$, John Stenos ${ }^{2}$, Robert L Rees ${ }^{3}$ and Rebecca J Traub ${ }^{1}$
}

\begin{abstract}
Background: The recent detection of Rickettsia felis DNA in dogs in Australia suggests that dogs are potential mammalian reservoir hosts for this emerging rickettsia. To date, there is no published report addressing the seroprevalence of $R$. felis in dogs in Australia.

Methods: Antigens for $R$. felis were produced by inoculating confluent XTC-2 monolayer cell cultures with three pools of cat flea (Ctenocephalides felis) homogenates. Infection was confirmed by real-time (qPCR), conventional or nested PCRs targeting the ompB, gltA, $17 \mathrm{kDa}$ and ompA genes. Two hundred and ninety-two dogs from Southeast Queensland and the Northern Territory were tested for the presence of $R$. felis antibodies using a microimmunofluorescence (IF) test and the seroprevalence and associated risk factors for exposure were determined using both uni- and multi-variate analyses.

Results: Rickettsia felis was successfully isolated in cell culture from all three cat-flea pools. One hundred and fortyeight dogs (50.7\%) showed seropositivity with titres $\geq 64$ and 54 (18.5\%) with titres $\geq 128$. At antibody titres $\geq 64$, dogs with active ectoparasite control were less likely to be seropositive to R. felis (OR: 2.60; 95\% Cl: 1.20 - 5.56).

Conclusions: This first reported isolation of $R$. felis in cell culture in Australia allowed for the production of antigen for serological testing of dogs. Results of this serological testing reflects the ubiquitous exposure of dogs to $R$. felis and advocate for owner vigilance with regards to ectoparasite control on domestic pets.
\end{abstract}

Keywords: Rickettsia felis, Flea-borne spotted fever, Seroprevalence, Ctenocephalides felis

\section{Background}

A number of rickettsial species are associated with human disease in Australia. These include Queensland tick typhus caused by $R$. australis, Flinders Island spotted fever caused by $R$. honei, Australian spotted fever by $R$. honei subspecies 'marmionii', epidemic typhus by $R$. prowazekii, murine typhus by $R$. typhi, scrub typhus by $O$. tsutsugamush $i$ and $\mathrm{Q}$ fever by Coxiella burnetti [1]. In recent years, the ubiquitous nature and potential veterinary public health significance of Rickettsia felis as an emerging rickettsial zoonosis that causes flea-borne spotted fever (FSF) has become increasingly apparent [2-6]. An increasing number of human cases have being reported worldwide, and in

\footnotetext{
*Correspondence: s.hii2@uq.edu.au

'Equal contributors

'School of Veterinary Science, The University of Queensland, Gatton,

Queensland 4343, Australia

Full list of author information is available at the end of the article
}

Australia the agent was reported for the first time affecting five household members ranging in age from 4-64 years, living with flea-ridden pets in Victoria, Australia [2].

The ubiquitous nature of $R$. felis and the risk it poses to human health is largely due to the global distribution of its biological vector, the 'cat flea' Ctenocephalides felis [5,7]. Infected cat fleas have been described in over 20 countries spanning five continents, with infection rates ranging from $15 \%$ in New Zealand [8] to $81 \%$ in New Caledonia [9]. In Australia, $19.8 \%$ of flea pools collected from cats in eastern Australia [10], 36\% from dogs and 33\% from cats in Western Australia [11], and 48.5\% from dogs in Southeast Queensland (SE QLD) and the Northern Territory (NT) (Hii et al., unpublished data) were demonstrated to carry R. felis DNA.

Although C. felis has been studied extensively and is a well-recognised biological vector for $R$. felis, surprisingly

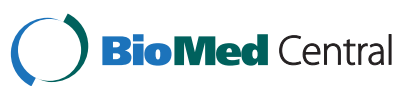


there is to date no consensus on the potential mammalian reservoir(s) for this emerging zoonosis. Several peridomestic species associated with the cat flea have been implicated, including cats, dogs, opossums and rats, all of which have been naturally seropositive or molecular positive for $R$. felis infection [3,12]. In Spain, 51.1\% of dogs had detectable antibodies to $R$. felis [13] supporting their role as potential reservoir hosts. On the other hand, a relatively low seroprevalence $(1.4 \%-13.1 \%)$, was documented in dogs from Brazil [14-16].

Recently, 9\% of pound dogs in SE QLD and 2.3\% of Indigenous community dogs in the NT, Australia were found to have detectable $R$. felis DNA in their blood, implying that domestic dogs were likely primary reservoir hosts for $R$. felis $[17,18]$. In these studies, all dogs appeared healthy, a common feature that is also usually a characteristic of reservoir hosts. To date seroepidemiological studies on rickettsial diseases involving dogs have focussed on their role as possible sentinel hosts for human rickettsioses in Australia. In 1991, 11.2\% of dogs from south eastern Australia, which included coastal New South Wales, eastern coastal Victoria, Flinders Island, and the Tasmanian mainland, were found to be seropositive to $R$. australis infection [19]. A serosurvey in Launceston, Tasmania, where spotted fever group (SFG) diseases are endemic, demonstrated that $57 \%$ of dogs had been exposed to SFG rickettsiae [20]. Recently, antibodies reactive with Coxiella burnetii were detected in $21.8 \%$ of domestic dogs from northern Queensland [21].

In this study, we isolated $R$. felis in cell culture to allow for the production of antigen for serological assays. We aimed to determine the seroprevalence and associated risk factors for exposure to $R$. felis in dogs from previously sampled regions in Queensland and the Northern Territory in order to support earlier findings suggesting that dogs were primary mammalian reservoir hosts for this agent.

\section{Methods}

\section{Sampling and PCR}

Single blood samples were collected into clotting tubes from a total of 292 dogs sourced from pounds, veterinary practices in SE QLD the NT and the Clinical Pathology Laboratory (CPL) based at the School of Veterinary Science, The University of Queensland. Sera was subsequently collected from clotting tubes and stored at $-80^{\circ} \mathrm{C}$ until analysed.

Pound dogs used for teaching purposes were sourced from the Clinical Studies Centre, School of Veterinary Science, The University of Queensland. Samples from client-owned dogs were sourced from five veterinary practices across SE QLD and one from Katherine in the NT. These dogs were presented to veterinary practices for many reasons including routine vaccination, neutering, heartworm testing, yearly health profiling and a range of illnesses. Blood and sera from the CPL were based on convenience; these samples were archived routine diagnostic specimens and would have otherwise been discarded. Following blinding for owner confidentiality, information with regards to age, sex, breed and ectoparasite control were recorded. This project was approved by the University of Queensland Animal Ethics Committee.

\section{Isolation of R. felis in cell culture}

Rickettsia felis antigen was isolated using XTC-2 cell lines, courtesy of the Australian Rickettsial Reference Laboratory, Geelong, Victoria. XTC-2 cell lines were cultured in $25 \mathrm{~cm}^{2}$ cell culture flasks with Leibowitz-15 (L-15) (GIBCO, Rockville, MD) medium supplemented with $5 \%(\mathrm{v} / \mathrm{v})$ foetal calf serum (Bovogen Biologicals, Australia), $2 \mathrm{mM}$ L-glutamine and L-amino-acids (GIBCO, Rockville, MD), and 1\% (v/v) tryptose phosphate (GIBCO) [22]. Cell lines were incubated at $28^{\circ} \mathrm{C}$ for 48-72 hours to obtain subconfluent cell monolayers.

Three pools of 20 live cat fleas, one collected from a pound dog in SE QLD and two from laboratory colonies maintained at the School of Veterinary Science, The University of Queensland were collected. These were surface sterilized by washing in $2 \%$ iodine for 3 minutes and $70 \%$ ethanol for 2 minutes, followed with a rinse in sterile distilled water. They were collected into $1.5 \mathrm{ml}$ centrifuge tubes containing $100 \mu \mathrm{l}$ culture medium and ground with sterile plastic pestles. One millilitre of culture medium containing $100 \mu \mathrm{g} / \mathrm{ml}$ gentamicin was added and the flea homogenate mixed. Five hundred microlitres of homogenate was transferred using a syringe filter (with a 0.45 $\mu \mathrm{m}$ membrane) into a $25 \mathrm{~cm}^{2}$ cell culture flask containing the XTC-2 monolayer cell lines with approximately $12 \mathrm{ml}$ of the antibiotic medium. The remaining homogenate was kept at $-20^{\circ} \mathrm{C}$ for PCR testing. The flasks were centrifuged at $250 \mathrm{~g}$ for 5 minutes at $20^{\circ} \mathrm{C}$. This was followed by a 24 hour incubation, after which, the media was replaced with antibiotic-free media. The inoculated cell lines were examined daily for contamination under a tissue culture microscope. The media was changed fortnightly and screened for rickettsial infection by Diff-Quick staining (Quick Dip, Fronine Lab Supplies, Australia), qPCR and conventional PCR.

DNA of flea homogenates and inoculated cell lines was extracted using the DNeasy Blood \& Tissue Kits (QIAGEN, Hilden, Germany) following the manufacturer's protocol. All extracted DNA of fleas and cell cultures were subjected to qPCR to detect the gltA gene according to the previous protocol [23], with some modification. Reactions were performed in a $10 \mu \mathrm{l} \mathrm{mix-}$ ture containing Kapa Probe Fast qPCR mastermix (Kapa Biosystems), $4 \mathrm{pmol}$ of each forward and reverse 
primers, 2 pmol of probe and $2 \mu \mathrm{l}$ of extracted DNA. All qPCR positive DNA samples were further analysed using a single rickettsiae-specific PCR targeting partial $o m p \mathrm{~B}$ and $17 \mathrm{kDA}$ genes, and a nested $R$. felis-specific PCR targeting gltA genes $[17,18,24]$ followed by bidirectional DNA sequencing to confirm rickettsial speciation.

In addition, an $R$. felis-specific PCR was developed to amplify a $1009 \mathrm{bp}$ of the ompA gene using newly designed primers - ompA-F1 5'-CGATAGTGTTACAAGTACCGG3' and ompA-R1 5'-GCATCTTCCATTAACTCAAGC-3'. PCRs were performed in a $25 \mu \mathrm{l}$ reaction mixture containing $2 \mu \mathrm{l}$ of DNA, $5 \mu \mathrm{l} 5 \mathrm{x}$ PCR buffer, $200 \mu \mathrm{mol} / \mathrm{L}$ dNTP, $2.0 \mathrm{mmol} / \mathrm{L} \mathrm{MgCl}_{2}, 0.5$ units of GoTaq polymerase (Promega, Madison, WI, USA), 10 pmol of each forward and reverse primer and a final volume of nuclease free water. PCRs were run at $95^{\circ} \mathrm{C}$ for $2 \mathrm{~min}$ for the initialization step, followed by 40 cycles of $95^{\circ} \mathrm{C}$ for $45 \mathrm{~s}, 57^{\circ} \mathrm{C}$ for $30 \mathrm{~s}$ and $72^{\circ} \mathrm{C}$ for $45 \mathrm{~s}$ with a final extension step of $72^{\circ} \mathrm{C}$ for $7 \mathrm{~min}$. All amplified PCR products were subjected to DNA sequencing.

\section{Preparation of IF test slides}

Rickettsia felis infected XTC-2 cell lines were harvested and inoculated into an uninfected monolayer of XTC-2 cell lines in the $25 \mathrm{~cm}^{2}$ cell culture flask. Cell lines were harvested when the infection rate of cells reached $90 \%$, as estimated by IF and Diff-Quick staining. The infected cells with medium were centrifuged at $500 \mathrm{~g}$ for $5 \mathrm{mi}$ nutes and the supernatant was discarded. The pellet was resuspended with sterile $1 \times$ PBS and heat inactivated at $56^{\circ} \mathrm{C}$ for 30 minutes. Two microlitres of the antigen was spotted onto each of the 40 well slides, air dried, and fixed in acetone for 10 minutes. Slides were kept at $4{ }^{\circ} \mathrm{C}$ until used.

\section{IF test}

An IF was performed following a previously described protocol $[20,25]$ with some modification. In brief, each serum sample was screened for $R$. felis antigen at 1:32 dilution in a $2 \%$ skimmed milk-PBS solution. All slides were incubated in a humid chamber at $37^{\circ} \mathrm{C}$ for 30 minutes, then washed with 1/10 PBS for 3 minutes and air-dried. Fluorescein isothiocyanate (FITC)-labelled goat anti-dog immunoglobulin G (Kirkegaard \& Perry Laboratories, USA) was added and slides were incubated, washed, airdried, mounted with fluorescence mounting medium (Dako, USA) and visualized under a UV microscope. Positive and negative dog sera were used as control in each reaction. Negative control serum was sourced from a dog previously tested to be non-reactive to $R$. felis, $R$. australis, $R$. honei, $R$. typhi, $R$. conorii and $R$. rickettsii. Positive control serum was sourced from a dog tested to be reactive only to $R$. felis independently by the Australian Rickettsial Reference Laboratory.
All sera showing a positive reaction at 1:32 were subjected to serial doubling dilution until an end-point was obtained. Discordant samples were read by a second examiner independently to confirm endpoint reactivity. Sera with titres of 1:64 or greater were considered positive, as previously described $[13,15,19]$.

\section{Statistics}

Statistical calculations were conducted using SPSS version 20.0 software (SPSS Inc., Chicago, IL, USA). The association between $R$. felis seropositivity (at titres $\geq 64$ and $\geq 128$ ) and putative risk factors (age, sex, breed, ectoparasite treatment status and ownership status) were evaluated in the univariable analysis using logistic regression models. Odds ratios and their 95\% confidence intervals were reported for each risk factor. Exact tests were used to evaluate the association of dichotomous risk factors with the presence of $R$. felis antibodies. Overall $P$-values for risk factors with more than two categories were assessed using joint-significance hypothesis tests.

After checking for collinearity, variables significant at $P \leq 0.2$ and with sufficient numbers $(\mathrm{n}>10)$ in the univariable analysis were considered eligible for inclusion in the multiple logistic regression analysis [26,27]. Backward elimination was used as a model building approach and risk factors were dropped from the multivariable model until all risk factors in the model were statistically significant at $\mathrm{P}<0.05$. [27].

\section{Results}

\section{Antigen production:}

Rickettsia felis was successfully isolated from all three inoculated XTC-2 cell lines as detected by qPCR, single $(o m p \mathrm{~B}$ and $17 \mathrm{kDa})$ and nested PCR $(g l t \mathrm{~A})$, and DiffQuick staining at 4 weeks post incubation. Partial ompA gene of $R$. felis was also amplified in all infected cell lines. The isolation of $R$. felis enabled the production of antigen for IF testing.

A total of 292 dog sera were collected from December 2009 to December 2012-185 from SE QLD and 107 from NT. Of these, 100 were pound dogs, 162 were clientowned dogs sourced from referral practices and 30 were convenience samples from the CPL. Of the CPL sourced samples, ownership status was confirmed in 18 dogs. In total, 180 dogs were client-owned.

There were 142 purebred dogs, 147 of mixed breed and 3 were of unknown breed. Most (66.9\%) dogs were adults (1-10 year), followed by young dogs $(<1$ year) $(20.2 \%)$ and geriatrics ( $>10$ years) $(12.9 \%)$. One hundred and forty three were male (49.7\%), 145 were female (50.3\%) and 4 were of unspecified sex. Ectoparasite control status was only available for 48 client-owned dogs. Of these, 42 dogs were subjected to ectoparasite control. 
Consultation with staff revealed that pound dogs had not received active ectoparasite control.

A total of $148 / 292(50.7 \%)$ and $54 / 292(18.5 \%)$ dogs were seropositive for $R$. felis with antibody titres of $\geq 64$ and $\geq 128$ respectively (Table 1 ). Of these, 94 had an antibody titre of 1:64, 42 an antibody titre of 1:128, 10 an antibody titre of 1:256, 1 an antibody titre of $1: 512$ and 1 an antibody titre of 1:8192.

Of the seven risk factors assessed in the univariable model, only ectoparasite prevention and desexing status were included in the multivariable logistic regression analysis. Cross-tabulation of desexing status at antibody titres of $\geq 64$ stratified for gender, indicated that 20/55 (36.4\%) of neutered females were seropositive for $R$. felis while $34 / 51$ (66.7\%) of intact females were seropositive $(P=0.002)$. This relationship was not significant for males $(\mathrm{P}=0.574)$. Hence, gender was forced into the multivariable model to explore the interaction between gender and desexing status. However, this interaction term was not significant in the multivariable model. The analysis revealed that dogs receiving no ectoparasite control (odds ratio 2.6, 95\% CI: $1.20-5.56, P=0.014$ ) were more likely to have antibodies to $R$. felis at titres of $\geq 64$. No risk factors were associated with $R$. felis antibody titres of $\geq 128$ at $\mathrm{P}<0.05$.

\section{Discussion}

This study represents the first isolation of $R$. felis in cell culture from cat fleas in Australia. This pathogenic agent is an obligate intracellular bacteria which requires nucleated eukaryotic cells to grow [28], and grows best at temperatures under $32^{\circ} \mathrm{C}$ [3]. XTC-2 cell lines are derived from Xenopus laevis, a South African clawed toad, which grows at $28^{\circ} \mathrm{C}$ and is suited to support the growth of $R$. felis at optimum levels. In contrast, the optimal

Table 1 Univariate analysis of risk factors and their association with $R$. felis seropositivity in dogs at antibody titres $\geq 64$ and $\geq 128$

\begin{tabular}{|c|c|c|c|c|c|c|c|}
\hline \multirow[t]{2}{*}{ Variable surveyed } & \multirow{2}{*}{$\begin{array}{c}\text { No of } \\
\text { sera } \\
\text { available }\end{array}$} & \multicolumn{3}{|c|}{ Antibody titre $\geq 64$} & \multicolumn{2}{|c|}{ Antibody titre $\geq 128$} & \multirow[b]{2}{*}{$P$ value } \\
\hline & & $\begin{array}{l}\text { No of seropositive } \\
\text { dogs }(\% ; 95 \% \mathrm{Cl})\end{array}$ & OR; $95 \% \mathrm{Cl}$ & $P$ value & $\begin{array}{l}\text { No of positive } \\
\text { dogs (\%) }\end{array}$ & OR; $95 \% \mathrm{Cl}$ & \\
\hline Total sera in the study & 292 & $148(50.7 \%)$ & $0.45-0.56$ & & $54(18.5 \%)$ & $0.14-0.23$ & \\
\hline Location & & & & 0.360 & & & 0.947 \\
\hline SE QLD dogs & 185 & $90(48.6 \%)$ & Reference & & $34(18.4 \%)$ & Reference & \\
\hline NT dogs & 107 & $58(54.2 \%)$ & $1.25 ; 0.78-2.01$ & & $20(18.7 \%)$ & $1.02 ; 0.55-1.88$ & \\
\hline Source & & & & 0.510 & & & 0.221 \\
\hline Client-owned & 180 & $88(48.9 \%)$ & Reference & & $29(16.1 \%)$ & Reference & \\
\hline Pound & 100 & $53(53.0 \% ;)$ & $1.18 ; 0.72-1.87$ & & $22(22 \%)$ & $1.47 ; 0.79,2.72$ & \\
\hline Status of active ectoparasite control & & & & 0.014 & & & 0.106 \\
\hline Active ectoparasite control & 42 & $12(28.6 \%)$ & Reference & & $4(9.5 \%)$ & Reference & \\
\hline No active ectoparasite control & 106 & $52(49.1 \%)$ & $2.60 ; 1.20-5.56$ & & $22(20.8 \%)$ & $2.49 ; 0.80,7.69$ & \\
\hline Breed & & & & 0.265 & & & 0.286 \\
\hline Purebred & 142 & 67 (47.2\%) & Reference & & $23(16.2 \%)$ & Reference & \\
\hline Mixed breed & 147 & 79 (53.7\%) & $1.30 ; 0.76-2.51$ & & $31(21.1 \%)$ & $1.38 ; 0.76,2.51$ & \\
\hline Age & & & & 0.298 & & & 0.128 \\
\hline Young (<1 year) & 58 & $25(43.1 \%)$ & Reference & & $7(12.7 \%)$ & Reference & \\
\hline Adult (1 - 10 year) & 192 & $101(52.6 \%)$ & $1.37 ; 0.76-2.46$ & & 41 (21.4\%) & $1.98 ; 0.84-4.69$ & 0.121 \\
\hline Geriatric (>10year) & 37 & $18(48.6)$ & $1.17 ; 0.51-2.67$ & & $4(10.8 \%)$ & $0.88 ; 0.24-3.25$ & 0.852 \\
\hline Gender & & & & 0.638 & & & 0.414 \\
\hline Male & 143 & 70 (49.0\%) & Reference & & $29(20.3 \%)$ & $1.28 ; 0.71,2.33$ & \\
\hline Female & 145 & $75(51.7 \%)$ & $1.12 ; 0.70-1.77$ & & $24(16.6 \%)$ & Reference & \\
\hline Desexing status & & & & 0.032 & & & 0.062 \\
\hline Neutered & 111 & 48 & Reference & & $16(14.4 \%)$ & Reference & \\
\hline Intact & 111 & 64 & $1.79 ; 1.05-3.04$ & & $27(24.3 \%)$ & $1.91 ; 0.96-3.78$ & \\
\hline
\end{tabular}

$\mathrm{Cl}$, confidence interval.

OR, Odds ratio. 
growing temperatures for typhus group $\left(35^{\circ} \mathrm{C}\right)$ and spotted fever group $\left(32^{\circ} \mathrm{C}\right)$ rickettsiae are higher $[29,30]$.

Previous isolation of $R$. felis in XTC-2 cell lines was attempted using the shell vial centrifugation technique [22]. This technique is sensitive and frequently utilised for isolation of agents from clinical specimens [31,32] that contain a low burden of microorganisms. However, it is laborious, requires expertise and is not suitable for downstream production of antigen for serological assays. In this study, conventional cell culture was carried out utilising cell culture flasks to enable production of $R$. felis antigens in large amounts. Rickettsia felis has also been reportedly successfully cultivated in vertebrate and arthropod cell lines, including Vero cells, L929, ISE6 and C6/36 [22,33-35].

Our study represents the first to provide serological evidence for $R$. felis exposure in dogs in Australia. The high seroprevalence $(50.7 \%)$ is in agreement with a study conducted in Spain, where $51.1 \%$ of dogs were reported as exposed to this agent [13]. The high seroprevalence of $R$. felis in dogs in the present study was not unexpected. The cat flea, $C$. felis, is known to be the most common ectoparasite and dominant flea infesting dogs in Australia and its wide geographical distribution across the country $[11,36]$ suggests that the seroprevalence of $R$. felis reported in this study could be a representation of most populated areas of Australia. We found no significant difference in seropositivity between dogs located in SE QLD and NT despite the variation in climate. This suggests that dogs from these two regions have been equally exposed to $R$. felis, which is in turn likely attributable to frequent exposure to cat fleas. However, flea infestation in dogs in the current study was not evaluated, hence an association with the presence of $R$. felis antibodies could not be confirmed.

Besides fleas, DNA of $R$. felis has also been isolated from the brown dog tick, Rhipicephalus sanguineus [37]. This tick is highly prevalent in dogs in the NT due to its preference for the humid warm tropics with relative humidity of $60 \%-90 \%$ and temperatures of $20^{\circ} \mathrm{C}-30^{\circ} \mathrm{C}$ [38]. Whether this tick species acts as a true biological vector as opposed to simply being an incidental mechanical vector remains uncertain at this time.

Serological cross-reactivity among Rickettsia spp is common. $R$. felis antibodies have been known to be more reactive to $R$. typhi from the typhus group, than to the spotted fever group $[2,7,39,40]$. Moreover, a recent serosurvey study in Spain showed dogs that were positive for $R$. felis antibodies did not necessarily cross-react with $R$. typhi, with prevalences of $9.7 \%$ and $51.1 \%$ respectively [13]. A seroepidemiological study of $R$. felis, $R$. typhi and $R$. conorii infection in humans in Spain also demonstrated low levels of cross-reaction between $R$. felis and R. typhi or R. conorii [41]. These findings might suggest the possibility of high specificity of $R$. felis serological tests.

The current study highlights the importance of flea control in pets by demonstrating a significant association between active ectoparasite control and the absence of $R$. felis exposure. Although it is not statistically significant in the multivariable model, intact female animals in the current study showed higher seroprevalence of $R$. felis compared to neutered dogs, suggestive of possible association with gonadal hormonal factors that might influence the outcome of an infection [42]. Sex-associated behaviour such as roaming in intact males may predispose them to wider exposure to fleas and the pathogens they carry. This phenomenon has been observed in a number of studies whereby neutering decreased the prevalence of both endoparasites and tick-borne diseases in dogs [42-45].

The high seroprevalence in dogs in the present study, the detection of $R$. felis DNA in dog blood [17] and high infection rates in cat fleas sourced from dogs [11] support the role of dogs as potential reservoir hosts for this zoonosis [46]. Previous studies have demonstrated infection with rickettsial spotted fever in humans positively associated with owning or contacting dogs $[47,48]$. In Spain, seropositivity was associated with humans who had contact with domestic animals compared to farm and wild animals [49]. A dog whose owners were infected with FSF was also found to be infected by the same agent [4]. This study further provides evidence of the risks this emerging zoonosis poses, especially to companion animal owners and their families.

\section{Conclusion}

This study reports the first isolation of $R$. felis from $C$. felis in cell culture in Australia. This study reflects the natural ubiquitous exposure of dogs to $R$. felis in tropical and subtropical parts of northern and eastern Australia and advocates for owner vigilance with regards to ectoparasite control on domestic pets.

\section{Abbreviations}

qPCR: Real-time Polymerase chain reaction; SE QLD: Southeast Queensland; NT: Northern Territory; FSF: flea-borne spotted fever; SFG: spotted fever group; CPL: Clinical Pathology Laboratory; IF: microimmunofluorescence test.

\section{Competing interests}

The authors declare that they have no competing interests.

\section{Authors' contributions}

SFH carried out the laboratory work, data analysis, intellectual interpretation and writing of the manuscript. MYA supervised the study, carried out the laboratory work, intellectual interpretation and critical revision of the manuscript for publication. RJT designed the study project, supervised the study, and was involved in intellectual interpretation and critical revision of the manuscript for publication. SRK supervised the study and was involved in intellectual interpretation and critical revision of the manuscript for 
publication. JS and RLR revised the article critically for important intellectual content. All authors read and approved the final version of the manuscript.

\section{Acknowledgment}

The authors thank Dr Stephen Graves (the Director and founder of Australian Rickettsial Reference Laboratory) and scientist officers in the Australian Rickettsial Reference Laboratory, Geelong, Victoria for the rickettsial cell culture and serological assay training; veterinarians from referral practice and hospitals in SE QLD and Katherine, NT for sera collection; Glen Coleman and Kim Jell for providing laboratory cat flea pools; Joerg Henning for statistical analysis; This study was funded by Bayer Animal Health Australia.

\section{Author details}

'School of Veterinary Science, The University of Queensland, Gatton, Queensland 4343, Australia. ${ }^{2}$ Australian Rickettsial Reference Laboratory, Geelong, Victoria 3220, Australia. ${ }^{3}$ Bayer Animal Health Tingalpa, Tingalpa, Queensland 4173, Australia.

Received: 31 January 2013 Accepted: 29 May 2013

Published: 3 June 2013

\section{Reference}

1. Graves S, Stenos J: Rickettsioses in Australia. In Rickettsiology and Rickettsial Diseases. Volume 1166. Edited by Hechemy KE, Brouqui P, Samuel JE, Raoult DA. Oxford: Blackwell Publishing; 2009:151-155. Annals of the New York Academy of Sciences.

2. Williams M, Izzard L, Graves SR, Stenos J, Kelly JJ: First probable Australian cases of human infection with Rickettsia felis (cat-flea typhus). Med J Australia 2010, 194:41-43.

3. Reif KE, Macaluso KR: Ecology of Rickettsia felis: A Review. J Med Entomol 2009, 46:723-736.

4. Oteo JA, Portillo A, Santibanez S, Blanco JR, Perez-Martinez L, Ibarra V: Cluster of cases of human Rickettsia felis infection from Southern Europe (Spain) diagnosed by PCR. J Clin Microbiol 2006, 44:2669-2671.

5. Perez-Osorio CE, Zavala-Velazquez JE, Leon JJA, Zavala-Castro JE: Rickettsia felis as emergent global threat for humans. Emerg Infect Dis 2008, 14:1019-1023.

6. Socolovschi C, Mediannikov O, Sokhna C, Tall A, Diatta G, Bassene H, Trape JF, Raoult D: Rickettsia felis-associated Uneruptive Fever, Senegal. Emerg Infect Dis 2010, 16:1140-1142.

7. Abdad MY, Stenos J, Graves S: Rickettsia felis, an emerging fleatransmitted human pathogen. Emerg Health Threats 2011, 4:7168.

8. Kelly PJ, Meads N, Theobald A, Fournier PE, Raoult D: Rickettsia felis, Bartonella henselae, and B. clarridgeiae, New Zealand. Emerg Infect Dis 2004, 10:967-968.

9. Mediannikov O, Cabre O, Qu F, Socolovschi C, Davoust B, Marie JL, Parola P, Raoult D: Rickettsia felis and Bartonella clarridgeiae in fleas from New Caledonia. Vector Borne Zoonotic Dis 2011, 11:181-183.

10. Barrs VR, Beatty JA, Wilson BJ, Evans N, Gowan R, Baral RM, Lingard AE, Perkovic G, Hawley JR, Lappin MR: Prevalence of Bartonella species, Rickettsia felis, haemoplasmas and the Ehrlichia group in the blood of cats and fleas in eastern Australia. Aust Vet J 2010, 88:160-165.

11. Schloderer D, Owen H, Clark P, Stenos J, Fenwick SG: Rickettsia felis in fleas, Western Australia. Emerg Infect Dis 2006, 12:841-843.

12. Abramowicz KF, Rood MP, Krueger L, Eremeeva ME: Urban Focus of Rickettsia typhi and Rickettsia felis in Los Angeles, California. Vector Borne Zoonotic Dis 2011, 11:979-984.

13. Nogueras MM, Pons I, Ortuno A, Segura F: Seroprevalence of Rickettsia typhi and Rickettsia felis in dogs from north-eastern Spain. Clin Microbiol Infect 2009, 15:237-238.

14. Labruna MB, Horta MC, Aguiar DM, Cavalcante GT, Pinter A, Gennari SM, Camargo LMA: Prevalence of Rickettsia infection in dogs from the urban and rural areas of Monte Negro municipality, Western Amazon, Brazil. Vector Borne Zoonotic Dis 2007, 7:249-255.

15. Fortes FS, Silveira I, Moraes-Filho J, Leite RV, Bonacim JE, Biondo AW, Labruna MB, Molento MB: Seroprevalence of Rickettsia bellii and Rickettsia felis in dogs, Sao Jose dos Pinhais, State of Parana, Brazil. Rev Bras Parasitol Vet 2010, 19:222-227.

16. Melo AL, Martins TF, Horta MC, Moraes-Filho J, Pacheco RC, Labruna MB, Aguiar DM: Seroprevalence and risk factors to Ehrlichia spp. and
Rickettsia spp. in dogs from the Pantanal Region of Mato Grosso State, Brazil. Ticks Tick Borne Dis 2011, 2:213-218.

17. Hii SF, Kopp SR, Abdad MY, Thompson MF, O'Leary CA, Rees RL, Traub RJ: Molecular Evidence Supports the Role of Dogs as Potential Reservoirs for Rickettsia felis. Vector Borne Zoonotic Dis 2011, 11:1007-1012.

18. Hii SF, Kopp SR, Thompson MF, O'Leary CA, Rees RL, Traub RJ: Molecular evidence of Rickettsia felis infection in dogs from northern territory, Australia. Parasit Vectors 2011, 4:198.

19. Sexton DJ, Banks J, Graves S, Hughes K, Dwyer B: Prevalence of antibodies to spotted-fever group rickettsiae in dogs from southeastern Australia. AmJTrop Med Hyg 1991, 45:243-248.

20. Izzard L, Cox E, Stenos J, Waterston M, Fenwick S, Graves S: Serological prevalence study of exposure of cats and dogs in Launceston, Tasmania, Australia to spotted fever group rickettsiae. Aust Vet J 2010, 88:29-31.

21. Cooper A, Hedlefs R, Ketheesan N, Govan B: Serological evidence of Coxiella burnetii infection in dogs in a regional centre. Aust Vet $J$ 2011, 89:385-387.

22. Raoult D, La Scola B, Enea M, Fournier PE, Roux V, Fenollar F, Galvao MAM, de Lamballerie X: A flea-associated Rickettsia pathogenic for humans. Emerg Infect Dis 2001, 7:73-81.

23. Stenos J, Graves SR, Unsworth NB: A highly sensitive and specific real-time PCR assay for the detection of spotted fever and typhus group Rickettsiae. AmJTrop Med Hyg 2005, 73:1083-1085.

24. Leitner M, Yitzhaki S, Rzotkiewicz S, Keysary A: Polymerase chain reactionbased diagnosis of Mediterranean spotted fever in serum and tissue samples. AmJTrop Med Hyg 2002, 67:166-169.

25. Graves SR, Dwyer BW, McColl D, McDade JE: Flinders Island spotted fever: a newly recognised endemic focus of tick typhus in Bass Strait. Part 2. Serological investigations. Med J Aust 1991, 154:99-104.

26. Frankena K, Graat E: Multivariate analysis: logistic regression. In Application of Quantitative Methods in Veterinary Epidemiology. Edited by Noordhuizen J, Frankena K, van der Hoofd CM, Graat E. Wageningen: Wageningen Pers; 2001:137-178.

27. Hosmer D, Lemeshow S: Applied logistic regression. New York: John Wiley \& Sons Editions; 1989.

28. Pacheco R, Rosa S, Richtzenhain L, Szabo MPJ, Labruna MB: Isolation of Rickettsia bellii from Amblyomma ovale and Amblyomma incisum ticks from southern Brazil. Revista Mvz Cordoba 2008, 13:1273-1279.

29. Fournier P, Raoult D, Raoult D, Parola P: Bacteriology, taxonomy, and phylogeny of Rickettsia. Infect Dis Therapy Ser 2007, 43:1.

30. Weiss E, Moulder J: The rickettsias and chlamydias. Bergey's manual of systematic bacteriology 1984, 1:687-739.

31. Angelakis E, Richet H, Rolain JM, La Scola B, Raoult D: Comparison of realtime quantitative PCR and culture for the diagnosis of emerging Rickettsioses. PLoS Negl Trop Dis 2012, 6:e1540.

32. Marrero M, Raoult D: Centrifugation-shell vial technique for rapid detection of Mediterranean spotted fever rickettsia in blood culture. AmJTrop Med Hyg 1989, 40:197-199.

33. Radulovic S, Higgins JA, Jaworski DC, Dasch GA, Azad AF: Isolation, cultivation, and partial characterization of the ELB agent associated with cat fleas. Infect Immun 1995, 63:4826-4829.

34. Pornwiroon W, Pourciau SS, Foil LD, Macaluso KR: Rickettsia felis from cat fleas: Isolation and culture in a tick-derived cell line. Appl Environ Microbiol 2006, 72:5589-5595.

35. Horta MC, Labruna MB, Durigon EL, Schumaker TTS: Isolation of Rickettsia felis in the mosquito cell line C6/36. Appl Environ Microbiol 2006, 72:1705-1707.

36. Slapeta J, King J, McDonell D, Malik R, Homer D, Hannan P, Emery D: The cat flea (Ctenocephalides $f$. felis) is the dominant flea on domestic dogs and cats in Australian veterinary practices. Vet Parasitol 2011, 180(3-4):383-388.

37. Oliveira KA, Oliveira LS, Dias CCA, Silva A, Almeida MR, Almada G, Bouyer DH, Galvao MAM, Mafra CL: Molecular identification of Rickettsia felis in ticks and fleas from an endemic area for Brazilian Spotted Fever. Mem Inst Oswaldo Cruz 2008, 103:191-194.

38. Abd Rani P: The epidemiology of canine vector-borne diseases in India. University of Queensland: School of Veterinary Science; 2012. PhD thesis.

39. Adams JR, Schmidtmann ET, Azad AF: Infection of colonized cat fleas, Ctenocephalides felis (Bouche'), with a rickettsia-like microorganism. AmJTrop Med Hyg 1990, 43:400-409.

40. Schriefer ME, Sacci JB, Dumler JS, Bullen MG, Azad AF: Identification of a novel rickettsial infection in a patient diagnosed with murine typhus. J Clin Microbiol 1994, 32:949-954. 
41. Bernabeu-Wittel M, del Toro MD, Nogueras MM, Muniain MA, Cardenosa $N$ Marquez FJ, Segura F, Pachon J: Seroepidemiological study of Rickettsia felis, Rickettsia typhi, and Rickettsia conorii infection among the population of southern Spain. Eur J Clin Microbiol Infect Dis 2006, 25:375-381.

42. Kirkpatrick CE: Epizootiology of endoparasitic infections in pet dogs and cats presented to a veterinary teaching hospital. Vet Parasitol 1988, 30:113-124.

43. Mohamed AS, Moore GE, Glickman LT: Prevalence of intestinal nematode parasitism among pet dogs in the United States (2003-2006). J Am Vet Med Assoc 2009, 234:631-637.

44. Inpankaew T, Traub R, Thompson RC, Sukthana Y: Canine parasitic zoonoses in Bangkok temples. Southeast Asian J Trop Med Public Health 2007, 38:247-255.

45. Abd Rani PAM, Irwin PJ, Coleman GT, Gatne M, Traub RJ: A survey of canine tick-borne diseases in India. Parasit Vectors 2011, 4:141.

46. Dobler G, Pfeffer M: Fleas as parasites of the family Canidae. Parasit Vectors 2011, 4:139.

47. Mumcuoglu KY, Frish K, Sarov B, Manor E, Gross E, Gat Z, Galun R: Ecological studies on the brown dog tick Rhipicephalus sanguineus (Acari: Ixodidae) in southern Israel and its relationship to spotted fever group rickettsiae. J Med Entomol 1993, 30:114-121.

48. Angerami RN, Camara M, Pacola MR, Rezende RC, Duarte RM, Nascimento EM, Colombo S, Santos FC, Leite RM, Katz G, Silva L: Features of Brazilian spotted fever in two different endemic areas in Brazil. Ticks Tick Borne Dis 2012, 3:346-348.

49. Nogueras MM, Cardenosa N, Sanfeliu I, Munoz T, Font B, Segura F: Serological evidence of infection with Rickettsia typhi and Rickettsia felis among the human population of Catalonia, in the northeast of Spain. AmJTrop Med Hyg 2006, 74:123-126.

doi:10.1186/1756-3305-6-159

Cite this article as: Hii et al:: Seroprevalence and risk factors for Rickettsia felis exposure in dogs from Southeast Queensland and the Northern Territory, Australia. Parasites \& Vectors 2013 6:159.

\section{Submit your next manuscript to BioMed Central and take full advantage of:}

- Convenient online submission

- Thorough peer review

- No space constraints or color figure charges

- Immediate publication on acceptance

- Inclusion in PubMed, CAS, Scopus and Google Scholar

- Research which is freely available for redistribution 